\title{
The Numerical Invariants concerning the Total Domination for Generalized Petersen Graphs
}

\author{
Taiyin Zhao, ${ }^{1}$ Gohar Ali $\mathbb{D}^{2},{ }^{2}$ Nabila Hameed, ${ }^{2}$ Syed Inayat Ali Shah, ${ }^{2}$ and Yu-Ming Chu $\mathbb{D}^{3,4}$ \\ ${ }^{1}$ School of Information and Software Engineering, University of Electronic Science and Technology of China, \\ Chengdu 610054, China \\ ${ }^{2}$ Department of Mathematics, Islamia College Peshawar, Peshawar, Pakistan \\ ${ }^{3}$ Department of Mathematics, Huzhou University, Huzhou 313000, China \\ ${ }^{4}$ Hunan Provincial Key Laboratory of Mathematical Modeling and Analysis in Engineering, \\ Changsha University of Science \& Technology, Changsha 410114, China
}

Correspondence should be addressed to Yu-Ming Chu; chuyuming@zjhu.edu.cn

Received 22 May 2020; Revised 20 September 2020; Accepted 14 October 2020; Published 24 October 2020

Academic Editor: Feng Feng

Copyright (c) 2020 Taiyin Zhao et al. This is an open access article distributed under the Creative Commons Attribution License, which permits unrestricted use, distribution, and reproduction in any medium, provided the original work is properly cited.

A subset $S$ of $V(G)$ is called a total dominating set of a graph $G$ if every vertex in $V(G)$ is adjacent to a vertex in $S$. The total domination number of a graph $G$ denoted by $\gamma_{t}(G)$ is the minimum cardinality of a total dominating set in $G$. The maximum order of a partition of $V(G)$ into total dominating sets of $G$ is called the total domatic number of $G$ and is denoted by $d_{t}(G)$. Domination in graphs has applications to several fields. Domination arises in facility location problems, where the number of facilities (e.g., hospitals and fire stations) is fixed, and one attempts to minimize the distance that a person needs to travel to get to the closest facility. In this paper, the numerical invariants concerning the total domination are studied for generalized Petersen graphs.

\section{Introduction}

Graph theory has been used to study various concepts of partition of the vertex set for the graph $G[1-3]$. The domination (total domination) problem for the graph was studied from 1950s onward, and domination (total domination) in graphs is said to be NP-complete problem [4]. Domination (total domination) sets are of practical interest in several areas of mathematics and other branches of science $[5,6]$. In wireless networks, dominating (total dominating) sets are used to find efficient routes within ad hoc mobile networks. In documents' summarization, domination (total domination) sets are used. Dominating (total dominating) sets are closely related to independent sets. Total dominating set is independent if and only if it is a maximal input set [7].

Moreover, in computer science, weighted graphs are widely used in the developments of data mining, software testing, image processing, communication networks, and information security [8-11].
In this paper, the numerical invariants concerning the total domination are studied for generalized Petersen graphs [12-16].

\section{Preliminaries}

Let $G=(V, E)$ be a simple, undirected, and finite graph with the vertex set $V(G)$ and the edge set $E(G)$.

The (open) neighborhood of a vertex $u \in V(G)$, denoted by $N(u)$, is the set of all vertices adjacent to $u$, i.e., $N(u)=\{v \in V(G): u v \in E(G)\}$. The closed neighborhood of $u$ is defined as $N[u]=\{u\} \cup N(u)$ [17-19]. For a set $S \subseteq V(G)$, its open neighborhood is the set $N(S)=\bigcup_{u \in S} N(u)$, and its closed neighborhood is the set $N[S]=N(S) \cup S$. A dominating set of a graph $G$, abbreviated as $D S$, is a set $S$ of vertices in $G$ such that every vertex in $V(G) \backslash S$ is adjacent to a vertex in $S$. The domination number of a graph $G$, denoted by $\gamma(G)$, is the minimum cardinality of a dominating set. A total dominating set of a graph $G$, abbreviated as TDS, is a set $S$ of vertices in $G$ such that every vertex in $V(G)$ is adjacent 
to a vertex in $S$. If no proper subset of $S$ is a TDS of $G$, then $S$ is a minimal TDS of $G$. Every graph without isolated vertices has a TDS. The total domination number of a graph $G$, denoted by $\gamma_{t}(G)$, is the minimum cardinality of a TDS. A TDS of $G$ of minimum cardinality is called a $\gamma_{t}(G)$-set.

The concept of total domination was introduced by Cockayne et al. [20]. For more information, see [21, 22].

Let $\tau(G)$ be the total number of $\gamma_{t}(G)$-sets. For each vertex $v \in V(G)$, we define the total domination value of $v$, denoted by $\operatorname{TDV}_{G}(v)$, to be the number of $\gamma_{t}(G)$-sets to which $v$ belongs. Evidently, $0 \leq \operatorname{TDV}_{G}(v) \leq \tau(G)$ for any $G$ and any $v \in V(G)$.

Generalized Petersen graphs are important classes of commonly used interconnection networks and have been intensively studied. The generalized Petersen graph $P(n, m)$, $n \geq 3$ and $1 \leq m \leq\lfloor(n-1) / 2\rfloor$, consists of an outer $n$-cycle $u_{1}, u_{2}, \ldots, u_{n}$, a set of $n$ spokes $u_{i} v_{i}, 1 \leq i \leq n$, and $n$ edges $v_{i} v_{i+m}, 1 \leq i \leq n$, with indices taken as modulo $n$, see Figure 1 . Thus, its vertex set is the union of $U=\left\{u_{1}, u_{2}, \ldots, u_{n}\right\}$ and $V=\left\{v_{1}, v_{2}, \ldots, v_{n}\right\}$. We say that the first set consists of $u$ vertices and the second one of $v$ vertices. By a $u$-path in $P(n, m)$, we mean a path whose vertices consist of just $u$-vertices. A $v$-path is defined similarly. The generalized Petersen graph $P(n, m)$ is shown in Figure 1 .

Investigation of the total number of total domatic sets and the total number of total dominating sets of the generalized Petersen graph is the sole aim and interest of writing this paper. Cao et al. in [23] studied the total domination number of generalized Petersen graphs and obtained the exact value of $\gamma_{t}(P(n, 2))$. For $k \geq 2$, they proved

$$
\gamma_{t}(P(n, 2))= \begin{cases}2 k, & \text { for } n=3 k, \\ 2 k+2, & \text { for } n=3 k+1, \\ 2 k+2, & \text { for } n=3 k+2\end{cases}
$$

In this paper, we determine the exact values for $\gamma_{t}(P(n, m))$ when $n \equiv 0(\bmod 3)$ and $m \neq 0(\bmod 3)$ and when $m=4,5$, and 7 . We also investigate total number of total dominating sets for these cases.

\section{Main Results}

The following result provides a trivial lower bound on the total domination number of a graph in terms of the maximum degree of the graph.

Theorem 1 (see [24]). If $G$ is a graph with no isolated vertex and maximum degree $\Delta(G)$, then

$$
\gamma_{t}(G) \geq\left\lceil\frac{|V(G)|}{\Delta(G)}\right\rceil .
$$

Theorem 2. Let $P(n, m)$ be a generalized Petersen graph with $n=3 k \geq 6, k \geq 2$. If $m \neq 0(\bmod 3)$, then

$$
\gamma_{t}(P(n, m))=\left\lceil\frac{2 n}{3}\right\rceil=2 k \text {. }
$$

Proof. Let $n=3 k \geq 6$. According to Theorem 1, we get

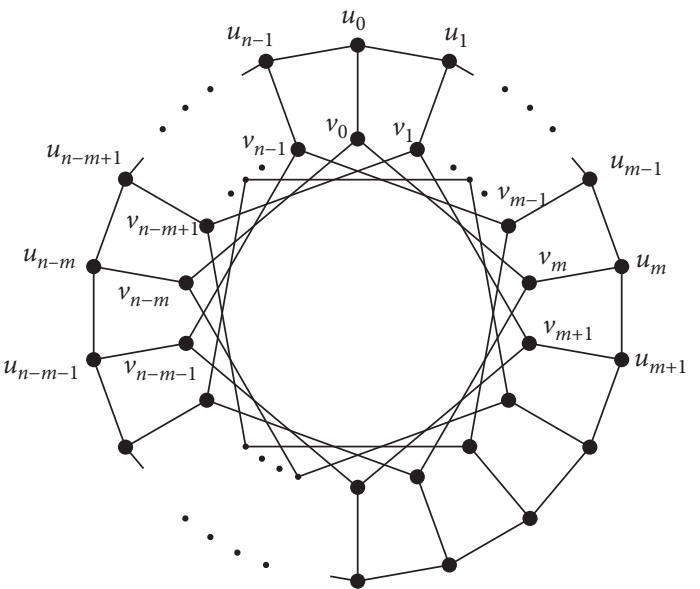

Figure 1: Generalized Petersen graph $P(n, m)$.

$$
\gamma_{t}(P(n, m)) \geq\left\lceil\frac{|V(P(3 k, m))|}{3}\right\rceil=\left\lceil\frac{6 k}{3}\right\rceil=2 k .
$$

Thus, to prove the equality, we just need to find a total dominating set of cardinality $2 k$. Let us consider a set

$$
S=\left\{u_{3 j+1}, v_{3 j+1}: j=0,1, \ldots, k-1\right\} .
$$

The vertex $u_{3 j+1}, j=0,1, \ldots, k-1$, dominates vertices $v_{3 j+1}, u_{3 j}$, and $u_{3 j+2}$, and the vertex $v_{3 j+1}, j=0,1, \ldots, k-1$, dominates vertices $u_{3 j+1}, v_{3 j+1+m}$, and $v_{3 j+1-m}$ with indices taken as modulo $n$. It is easy to see that, as $m \neq 0(\bmod 3)$, all vertices in $P(3 k, m)$ are dominated. Thus, $S$ is a total dominating set, and $|S|=2 k$. This concludes the proof.

Now, we proceed for $m=4$.

Theorem 3. Let $P(n, 4)$ be a generalized Petersen graph; then,

$$
\gamma_{t}(P(n, 4))= \begin{cases}2 k, & \text { for } n=3 k, k \geq 2, \\ 2 k+4, & \text { for } n=3 k+1, k \geq 7, \\ 2 k+4, & \text { for } n=3 k+2, k \geq 7 .\end{cases}
$$

Proof. Let us consider three cases:

Case 1: when $n=3 k, k \geq 2$, the result follows from Theorem 2.

Case 2: when $n=3 k+1, k \geq 7$, without loss of generality, consider a set $S_{0}=\left\{u_{3 j+1}, v_{3 j+1}: j=0,1, \ldots, k\right\}$. The vertex $u_{3 j+1}, j=0,1, \ldots, q$, dominates vertices $v_{3 j+1}, u_{3 j}$, and $u_{3 j+2}$, and the vertex $v_{3 j+1}, j=0,1, \ldots, q$, dominates vertices $u_{3 j+1}, v_{3 j+4}$, and $v_{3 j-3}$ with indices taken as modulo $n$. Thus, the vertices from this set $S_{0}$ dominate all but two vertices in $G$. The two vertices which are not dominated are $v_{2}$ and $v_{n-1}$. To totally dominate $v_{2}$ and $v_{n-1}$, we add their neighbors $u_{2}$ and $u_{n-2}$ to $S_{0}$ because these two vertices are already adjacent vertices in $S_{0}$. This means that the set $S=S_{0} \cup\left\{u_{2}, u_{n-2}\right\}$ totally dominates all vertices in $G$. Thus, $\gamma_{t}(P(3 k+1,4)) \leq|S|=2 k+4$. 
For necessity, we need to prove the reverse inequality, i.e., $\gamma_{t}(P(3 k+1,4)) \geq 2 k+4$. The proof will be by mathematical induction. For $n=22$, the result is obvious.

Since the result is true for all $P(n, 4)$ of the number of vertices less than $n$ when $m \equiv 0(\bmod 3)$, for $n \equiv 1(\bmod 3)$, let $D$ be any total dominating set of $P(n, 4)$. Then, $D=D_{1} \cup\left\{u_{2}\right\} \cup\left\{u_{n-2}\right\} \cup\left\{u_{n}\right\} \cup\left\{v_{n}\right\}$, where $D_{1}$ is the total dominating set of $P(n, 4)$ if only $n \equiv 0(\bmod 3)$, and we get $\left|D_{1}\right| \geq\lceil(2(n-2)) / 3\rceil=2 k$. Thus, we proved our required assertion.

Case 3: when $n=3 k+2, k \geq 4$, without loss of generality, consider a set $S_{0}=\left\{u_{3 j+1}, v_{3 j+1}: j=0,1, \ldots, k\right\}$. The vertex $u_{3 j+1}, j=0,1, \ldots, q$, dominates vertices $v_{3 j+1}, u_{3 j}$, and $u_{3 j+2}$, and the vertex $v_{3 j+1}, j=0,1, \ldots, q$, dominates vertices $u_{3 j+1}, v_{3 j+4}$, and $v_{3 j-3}$ with indices taken as modulo $n$. Thus, the vertices from this set $S_{0}$ dominate all but two vertices in $G$. The two vertices which are not dominated are $v_{2}$ and $v_{n-2}$. To totally dominate $v_{2}$ and $v_{n-2}$, we add these two vertices to $S_{0}$ because these two vertices are adjacent. This means that the set $S=S_{0} \cup\left\{v_{2}, v_{n-2}\right\}$ totally dominates all vertices in $G$. Thus, $\gamma_{t}(P(3 k+2,4)) \leq|S|=2 k+4$.

For necessity, we need to prove the reverse inequality, i.e., $\gamma_{t}(P(3 k+2,4)) \geq 2 k+4$. The proof will be by mathematical induction.

Assume the result is true for all $P(n, 4)$ of the number of vertices less than $n$. For $n \equiv 2(\bmod 3)$, let $D$ be any total dominating set of $P(n, 4)$. Then, $D=D_{1} \cup\left\{u_{2}\right\} \cup\left\{u_{n-2}\right\} \cup$ $\left\{u_{n-1}\right\} \cup\left\{v_{n-1}\right\}$, where $D_{1}$ is the total dominating set of $P(n, 4)$ if only $n \equiv 0(\bmod 3)$, and then $\left|D_{1}\right| \geq\lceil(2(n-2)) / 3\rceil=$ $2 k$, i.e., $|D| \geq 2 k+4$.

Theorem 4. Let $P(n, m)$ be a generalized Petersen graph. Then, $d_{t}(P(n, m))=3$ if $n \equiv 0(\bmod 3)$ and $m \neq 3 q$.

Proof. Let $\left\{P_{1}, P_{2}, P_{3}\right\}$ be the total domatic partitions since no vertex of any partition is contiguous to any other partitions and all the three domatic partitions totally dominate $G(P(n, m))$. Also, we know that $\operatorname{TDV}\left(u_{i}\right)=\operatorname{TDV}\left(v_{i}\right)=1$. We proved in Theorem 6 in the following that $\tau(P(n, m))=$ 3 if only $n \equiv 0(\bmod 3)$. It is observed that there is no recurrence in the total number of total dominating sets. Hence, we can write $d_{t}(P(n, m))=\tau(P(n, m))=3$ if only $n \equiv 0$ $(\bmod 3)$.

Henning and Yeo [24] proved the following.

Theorem 5 (see [24]). For any connected graph G,

$$
\sum_{v \in V(G)} \operatorname{TDV}_{G}(v)=\tau(G) \cdot \gamma_{t}(G)
$$

Theorem 6. Let $n \neq 10$ and $m \geq 4$ be positive integers. If $m \neq 0(\bmod 3)$, then

$$
\tau(P(n, m))= \begin{cases}3, & \text { for } n \equiv 0(\bmod 3), \\ n, & \text { for } n \equiv 0(\bmod 3),\end{cases}
$$

where for $m=5, n$ must be odd.

Proof. Let $m \geq 4$ be a positive integer, $m \neq 0(\bmod 3)$.

Case 1: when $n=3 k, k \geq 2$, then $\gamma_{t}(P(n, m))=2 k$, and a $\gamma_{t}(P(n, m))$-set $\Gamma$ comprises $k P_{2}$ 's, and $\Gamma$ is fixed by the choice of the first $P_{2}$. There is exactly one $\gamma_{t}(P(n, m))$-set comprising vertices $u_{1}$ and $v_{1}$. Thus, $\operatorname{TDV}\left(u_{1}\right)=\operatorname{TDV}\left(v_{1}\right)=1$. Similarly, $\operatorname{TDV}\left(u_{2}\right)=$ $\operatorname{TDV}\left(v_{2}\right)=1, \ldots, \operatorname{TDV}\left(u_{n}\right)=\operatorname{TDV}\left(v_{n}\right)=1$. Using Theorem 5, we get

$$
\begin{aligned}
\sum_{i=1}^{2 n} 1 & =2 k \cdot \tau(P(n, m)), \\
2 n \cdot 1 & =2 k \cdot \tau(P(n, m)) .
\end{aligned}
$$

Hence, $\tau(P(n, m))=3$ in this case.

Case 2: when $n=3 k+1$ or $n=3 k+2$, then a $\gamma_{t}(P(n, m))$-set $\Gamma$ is composed of in exactly one of the following ways:

(1) $\Gamma$ comprises $k P_{2}$ 's and one $P_{4}$

(2) $\Gamma$ comprises $(k-1) P_{2}$ 's and two $P_{3}$ 's

(3) $\Gamma$ comprises $(k-1) P_{2}$ 's and one $P_{4}$

(4) $\Gamma$ comprises $(k-1) P_{2}$ 's and two $P_{4}$ 's

(5) $\Gamma$ comprises $(k+1) P_{2}$ 's

Now, we discuss these subcases.

(i) Subcase 1: when $\langle\Gamma\rangle \equiv k P_{2} \cup P_{4}$, note that $\Gamma$ is fixed by the choice of single $P_{4}$. Selecting $P_{4}$ is the same as selecting its initial vertex in the counterclockwise order; thus, $\tau(P(n, m))=n$.

(ii) Subcase 2: when $\langle\Gamma\rangle \equiv(k-1) P_{2} \cup 2 P_{3}$, the set $\Gamma$ is fixed by the choice of two $P_{3}$ 's, and there are $n$ options for choosing the first two $P_{3}$ 's; thus, $\tau(P(n, m))=n$.

(iii) Subcase 3: when $\langle\Gamma\rangle \equiv(k+1) P_{2} \cup P_{4}$, there is only one chance for choosing single $P_{4}$ and $(k+1)$ chances for choosing $P_{2}$. Each vertex has equal number of chances. Each vertex is equally repeated in the total number of total dominating sets three times; thus, the total number of total dominating sets will be $n$.

(iv) Subcase 4: when $\langle\Gamma\rangle \equiv(k-1) P_{2} \cup 2 P_{4}$, then $\Gamma$ is fixed by the choice of double $P_{4}$. Each vertex is repeated three times in the total number of dominating sets, and for each vertex, there are equal number of chances for total domination; hence, each TDS will be repeated $n$ times.

(v) Subcase 5: when $\langle\Gamma\rangle \equiv(k+1) P_{2}$, there are exactly two vertices in each $P_{2}$. Each vertex of $P_{2}$ dominates three vertices including the vertex itself because this is a 3-regular graph. Thus, there are $n$ number of choices for the total number of dominating sets; thus, in all cases, $\tau(P(n, m))=n$. 
Corollary 1. Let $P(n, 5)$ be a generalized Petersen graph; then,

$$
\gamma_{t}(P(n, 5))= \begin{cases}4 k+4, & \text { for } n=6 k+1, k \geq 2, \\ 4 k+4, & \text { for } n=6 k+5, k \geq 1 .\end{cases}
$$

Proof. The proof is similar to the proof of Theorem 3.

Corollary 2. Let $P(n, 7)$ be a generalized Petersen graph; then,

$$
\gamma_{t}(P(n, 7))= \begin{cases}2 k+6, & \text { for } n=3 k+1, k \geq 5 \\ 2 k+6, & \text { for } n=3 k+2, k \geq 5\end{cases}
$$

Proof. The proof is similar to the proof of Theorem 3.

\section{Total Domatic Number of a Graph}

The total domatic number of a graph $G$ is denoted by $d_{t}(G)$ and is defined as $d_{t}(G)=s$, where $V(G)=\bigcup_{i=1}^{s} D_{i}$ and $D_{i}^{\prime} s$ are the total dominating sets and are the maximum disjoint partition of the vertex set of the graph.

Proposition 1. For any simple graph $G$ with minimum degree $\delta(G)$, the total domatic number $d_{t}(G) \leq \delta(G)$.

In the following result, our proof is simple from the proof of [25].

Theorem 7 (see [25]). For a graph $G$ of order $n$ and minimum degree $\delta(G)$, the lower bound for the total domatic number $\quad d_{t}(G) \geq\lfloor n /(n-\delta(G)+1)\rfloor, \quad$ where $n-\delta(G)+1=d_{t}(G)$.

Proof. Let $D$ be the total dominating set of the graph $G$ and $G^{c}$ be the complement of $G$. If $\delta(G)$ is the minimum degree of $G$, then $n-\delta(G)-1$ is the maximum degree of $G^{c}$, and due to this, any vertex $x$ of the graph $G$ is adjacent to at most $n-\delta(G)-1$ vertices of $D$ in $G^{c}$. If the vertex $x \in D$, then a vertex $z$ of the graph exists which is not adjacent to $x$ in $G^{c}$ but $x z \in E(G)$; this confirms that $|D| \geq n-\delta(G)+1$, and hence, $d_{t}(G) \geq\lfloor n /(n-\delta(G)+1)\rfloor$.

Corollary 3. For $G=P(n, m)$ generalized Petersen graph of order $2 n$ and $\delta(G)=3$, we have $1 \leq d_{t}(G) \leq 3$.

\section{Open Problems}

We conclude the paper with the following open problems for further investigation.

Open Problem 1. Characterize $\gamma_{t}(P(n, m))$ for all feasible values of $n$ and $m$.

Open Problem 2. Characterize $\operatorname{TDV}(u)$ for each $u \in V(P(n, m))$.
5.1. Discussion. In this paper, the generalized Petersen graph $(P(m, n))$ is investigated for the total domination number $\gamma_{t}$, total number of dominating sets $\tau$, and total domatic number $d_{t}(P(n, m))$. Here, we found the total dominating number for some restricted values of $m \in \mathbb{Z}^{+}$. For detailed applications of our results, refer [26-28].

\section{Data Availability}

All data required for this paper are included within this paper.

\section{Conflicts of Interest}

The authors do not have any conflicts of interest.

\section{Authors' Contributions}

Taiyin Zhao gave applications of results and improved the presentation of the paper, Gohar Ali supervised this work, Nabila Hameed proved the results, Syed Inayat Ali Shah wrote the first version of the paper, and Yu-Ming Chu wrote the final version of the paper and arranged funding for this paper.

\section{Acknowledgments}

This research was supported by the National Natural Science Foundation of China (Grant nos. 11971142, 11871202, $61673169,11701176,11626101$, and 11601485).

\section{References}

[1] F. Aslam, Z. Zahid, S. Zahid, and S. Zafar, "3-total edge mean cordial labeling of some standard graphs," Open Journal of Mathematical Sciences, vol. 3, no. 1, pp. 129-138, 2019.

[2] F. Asif, Z. Zahid, and S. Zafar, "Leap Zagreb and leap hyperZagreb indices of Jahangir and Jahangir derived graphs," Engineering and Applied Science Letter, vol. 3, no. 2, pp. 1-8, 2020.

[3] S. M. Kang, M. A. Zahid, A. u. R. Virk, W. Nazeer, and W. Gao, "Calculating the degree-based topological indices of dendrimers," Open Chemistry, vol. 16, no. 1, pp. 681-688, 2018.

[4] M. A. Henning, "Graphs with large total domination number," Journal of Graph Theory, vol. 35, no. 1, pp. 21-45, 2000.

[5] S. Gravier, "Total domination number of grid graphs," Discrete Applied Mathematics, vol. 121, no. 1-3, pp. 119-128, 2002.

[6] M. A. Henning and D. F. Rall, "On the total domination number of Cartesian products of graphs," Graphs and Combinatorics, vol. 21, no. 1, pp. 63-69, 2005.

[7] M. Chellali and T. W. Haynes, "A note on the total domination number of a tree," Journal of Combinatorial Mathematics and Combinatorial Computing, vol. 58, p. 189, 2006.

[8] W. Gao, M. Younas, A. Farooq, A. Virk, and W. Nazeer, "Some reverse degree-based topological indices and polynomials of dendrimers," Mathematics, vol. 6, no. 10, p. 214, 2018.

[9] Y. C. Kwun, A. Ali, W. Nazeer, M. Ahmad Chaudhary, and S. M. Kang, "M-polynomials and degree-based topological indices of triangular, hourglass, and jagged-rectangle 
benzenoid systems," Journal of Chemistry, vol. 2018, Article ID 8213950, 8 pages, 2018.

[10] Y. Kwun, A. Virk, W. Nazeer, M. Rehman, and S. Kang, "On the multiplicative degree-based topological indices of Siliconcarbon $\mathrm{Si}_{2} \mathrm{C}_{3}$-I [p, q] and Si2C3-II [p, q]," Symmetry, vol. 10, no. 8, p. 320, 2018.

[11] M. A. Umar, "Cyclic-antimagic construction of ladders," Engineering and Applied Science Letters, vol. 2, no. 2, pp. 43-47, 2019.

[12] F. Zhang, S. Nazeer, M. Habib, T. J. Zia, and Z. Ren, "Radio number for generalized Petersen graphs $\$ \mathrm{P}(n, 2)$," IEEE Access, vol. 7, pp. 142000-142008, 2019.

[13] S. M. Kang, S. Nazeer, W. Nazeer, and A. Rafiq, "Multilevel distance labeling for generalized Petersen $P(4 \mathrm{k}+2,2)$ related graphs," International Journal of Mathematical Analysis, vol. 8, pp. 1027-1039, 2014.

[14] G. Xu, "2-rainbow domination in generalized Petersen graphs P (n, 3)," Discrete Applied Mathematics, vol. 157, no. 11, pp. 2570-2573, 2009.

[15] R. Nedela and M. Škoviera, "Which generalized Petersen graphs are cayley graphs?" Journal of Graph Theory, vol. 19, no. 1, pp. 1-11, 1995.

[16] M. E. Watkins, "A theorem on tait colorings with an application to the generalized Petersen graphs," Journal of Combinatorial Theory, vol. 6, no. 2, pp. 152-164, 1969.

[17] J.-B. Liu, M. Younas, M. Habib, M. Yousaf, and W. Nazeer, "M-Polynomials and degree-based topological indices of VC5C7 [p, q] and HC5C7 [p, q] nanotubes," IEEE Access, vol. 7, pp. 41125-41132, 2019.

[18] W. Nazeer, A. Farooq, M. Younas, M. Munir, and S. Kang, "On molecular descriptors of carbon nanocones," Biomolecules, vol. 8, no. 3, p. 92, 2018.

[19] A. Farooq, M. Habib, A. Mahboob, W. Nazeer, and S. M. Kang, "Zagreb polynomials and redefined zagreb indices of dendrimers and polyomino chains," Open Chemistry, vol. 17, no. 1, pp. 1374-1381, 2019.

[20] E. J. Cockayne, R. M. Dawes, and S. T. Hedetniemi, "Total domination in graphs," Networks, vol. 10, no. 3, pp. 211-219, 1980.

[21] T. W. Haynes, S. T. Hedetniemi, and P. J. Slater, Fundamentals of Domination in Graphs, Marcel Dekker. Inc., New York, NY, USA, 1998.

[22] A. A. Bertossi, "Dominating sets for split and bipartite graphs," Information Processing Letters, vol. 19, no. 1, pp. 37-40, 1984.

[23] J. Cao, W. Lin, and M. Shi, "Total domination number of generalized Petersen graphs," Intelligent Information Management, vol. 1, no. 1, pp. 14-17, 2009.

[24] M. A. Henning and A. Yeo, Total Domination in Graphs, Springer, Berlin, Germany, 2013.

[25] H. Aram, S. M. Sheikholeslami, and L. Volkmann, "On the total domatic number of regular graphs," Transactions on Combinatorics, vol. 1, no. 1, pp. 45-51, 2012.

[26] H. F. M. Salih, S. M. Mershkhan, and S. M. Mershkhan, "Generalized the Liouville's and Möbius functions of graph," Open Journal of Mathematical Sciences, vol. 4, no. 1, pp. 186-194, 2020.

[27] A. Shah and S. A. U. H. Bokhary, "On chromatic polynomial of certain families of dendrimer graphs," Open Journal of Mathematical Sciences, vol. 3, no. 1, pp. 404-416, 2019.

[28] Y. J. Ge, J. B. Liu, M. Younas, M. Yousaf, and W. Nazeer, "Analysis of and nanotubes via topological indices," Journal of Nanomaterials, vol. 2019, Article ID 2072789, 10 pages, 2019. 